\title{
Survival After Lung Transplantation of Cystic Fibrosis Patients Infected with Burkholderia cepacia Complex
}

\author{
B. D. Alexander ${ }^{a}, *$, E. W. Petzold ${ }^{b}$, L. B. Reller ${ }^{a}$, \\ S. M. Palmer' ${ }^{\text {, R. D. Davis }}{ }^{\text {d }}$, C. W. Woods ${ }^{a}$ \\ and J. J. LiPuma ${ }^{e}$ \\ a Division of Infectious Diseases and International Health, \\ ${ }^{\mathrm{b}}$ Duke Human Vaccine Institute, ${ }^{\mathrm{C}}$ Division of Pulmonary \\ and Critical Care Medicine, Department of Medicine and \\ ¿Division of Cardiovascular and Thoracic Surgery, \\ Department of Surgery, Duke University School of \\ Medicine, Durham, NC \\ e Department of Pediatrics and Communicable Diseases, \\ University of Michigan Medical School, Ann Arbor, MI \\ * Corresponding author: Barbara D. Alexander, \\ alexa011@mc.duke.edu
}

Within the Burkholderia cepacia complex (Bcc), B. cenocepacia portends increased mortality compared with other species. We investigated the impact of Bcc infection on mortality and re-infection following lung transplant (LT). Species designation for isolates from Bcc-infected patients was determined using 16S rDNA and recA gene analyses. Of 75 cystic fibrosis patients undergoing LT from September 1992 to August 2002, 59 had no Bcc and 16 had Bcc (including 7 B. cenocepacia) isolated in the year before LT. Of the latter, $87.5 \%$ had Bcc recovered after transplantation, and all retained their pretransplant strains. Survival was $\mathbf{9 7 \%}$, $92 \%, 76 \%$ and $63 \%$ for noninfected patients; $89 \%, 89 \%$, $67 \%$ and $56 \%$ for patients infected with Bcc species other than B. cenocepacia; and $71 \%, 29 \%, 29 \%$ and $29 \%$ for patients with $B$. cenocepacia $(p=0.014)$ at 1 month, 1 year, 3 years and 5 years, respectively. Patients infected with $B$. cenocepacia before transplant were six times more likely to die within 1 year of transplant than those infected with other Bcc species $(p=$ 0.04 ) and eight times than noninfected patients ( $p<$ 0.00005). Following LT, infection with Bcc species other than $B$. cenocepacia does not significantly impact 5year survival whereas infection with $B$. cenocepacia pretransplant is associated with decreased survival.

Key words: Bacterial infection, lung transplantation, transplant infectious diseases, transplant outcome

Received 16 August 2007, revised 4 December 2007 and accepted for publication 18 January 2008

\begin{abstract}
Introduction
The Burkholderia cepacia complex (Bcc) consists of at least nine phenotypically similar but genotypically distinct species (previously referred to as genomovars) of nonfermenting, gram-negative rods. These include $B$. cepacia (genomovar I), B. multivorans (genomovar II), B. cenocepacia (genomovar III), B. stabilis (genomovar IV), B. vietnamiensis (genomovar V), $B$. dolosa (genomovar VI), $B$. ambifaria (genomovar VII), B. anthina (genomovar VIII) and $B$. pyrrocinia (genomovar IX) (1-4). Colonization and subsequent infection in cystic fibrosis (CF) patients with Bcc has been associated with poor outcomes, including accelerated decline in pulmonary function and fatal disease (57). Organisms belonging to each Bcc species have been recovered from patients with $C F$; however, the majority of clinical isolates are B. multivorans and B. cenocepacia (811). Furthermore, isolates associated with increased morbidity and mortality may cluster within the $B$. cenocepacia species $(12,13)$. Several epidemiologic studies have shown that some strains of Bcc are highly transmissible between patients, although a few studies have shown no evidence of transmission (14). Based on these observations, it seems that certain Bcc species and / or strains may be more transmissible than others, and likewise, some may be more virulent.
\end{abstract}

Lung transplantation is a therapeutic option for an increasing number of persons affected by CF. Patients with CF who are infected with Bcc present a particular dilemma for lung transplantation. Aris et al. reported in a retrospective review of patients undergoing lung transplantation that mortality was significantly increased in patients infected with Bcc (15). Snell and colleagues had previously reported a similar finding of high mortality (47\%) among patients infected with Bcc prior to transplant (16). In a series of $53 \mathrm{CF}$ patients transplanted in Toronto between 1988 and 1996, 1 -year survival was $67 \%$ in the Bcc-infected patients versus $92 \%$ among noninfected patients. A majority $(9 / 15$, $60 \%$ ) of deaths in the Bcc cohort was in the first 3 months following transplantation and was characterized by progressive acute respiratory distress syndrome and sepsis (17). Consequently, many centers consider infection with Bcc a contraindication to transplantation. Other centers, however, continued to transplant these patients. In support of this practice, Kanj et al. reported the early experience at Duke University Medical Center (DUMC), where infection with Burkholderia species did not seem to predict a worse 
outcome (18). Likewise, Flume and Egan reported lower mortalities associated with Bcc infection than previously described $(19,20)$. More recent studies suggest that within the Bcc, B. cenocepacia is associated with increased mortality rates compared with other species (21-23). For example, of $121 \mathrm{CF}$ patients transplanted at the University of North Carolina, excessive early mortality was noted in Bccinfected patients, with a 6-month mortality of 33\% compared with $12 \%$ in noninfected patients. All early deaths occurred in patients infected with B. cenocepacia (22).

DUMC is a CF treatment and lung transplantation center. The first lung transplant (LT) procedure at DUMC was performed in 1992, and until 2002, patients infected with Bcc including $B$. cenocepacia were regularly transplanted at DUMC. This retrospective investigation was performed to assess the impact of Bcc infection on mortality and reinfection following lung transplantation in patients with $\mathrm{CF}$.

\section{Materials and Methods}

Following Duke University Health System Institutional Review Board approval, the DUMC LT database was queried for all patients undergoing transplantation between September 1992 and August 2002 and consecutive patients with $C F$ as the underlying disease leading to $L T$ were thus identified and included in the study. Using a standardized case report form, clinical data were collected from paper charts and computerized records. Recipient age at time of transplant, donor age, mechanical ventilation for recipient at time of $L T$, graft ischemia time, mismatches in human leukocyte antigens, donor/recipient CMV serostatus and date of death and/or lung retransplantation were recorded. Complete 5 year follow-up was available on each patient. Prior to transplant, respiratory samples were submitted for bacterial culture that included Bcc selective media (OFPBL Agar, Remel, Lenexa, KS), as part of routine work-up for transplantation and for management of $\mathrm{CF}$ including acute exacerbations. Patients underwent surveillance bronchoscopy with bronchoalveolar lavage (BAL) bacterial culture at 1, 3, 6,9 and 12 months posttransplant or at any point if clinical indications developed (e.g. decrease in lung function, fever, new infiltrate on chest radiograph). Sputum samples for diagnostic purposes were also submitted from patients with clinical symptoms who were able to produce sputum.

Bcc were defined as multidrug resistant if they were intermediate or resistant to at least one agent in each of three classes of antibiotics including the $\beta$-lactams, sulfonamides and fluoroquinolones. Organisms were defined as panresistant if they were resistant to all drugs tested. LT recipients infected with Bcc generally received four systemic antibiotics known to be effective against the patient's Bcc isolate based on susceptibility testing for at least 3 weeks. For isolates found to be multidrug or panresistant, antibiotics were selected based on two-drug combination synergy testing performed by Dr. Lisa Saiman's laboratory, Columbia University. All CF patients transplanted at DUMC also received aerosolized colistin and tobramycin for 3 months following transplant as routine prophylaxis, regardless of infecting flora. Patients received triple drug immunosuppression with cyclosporine (prior to 1999) or tacrolimus (after 1999), azathioprine and corticosteroids. Induction with an interleukin-2 receptor agent (basiliximab) was also administered beginning in 1999. Details of the surgical procedure, posttransplant management and follow-up have been previously described $(24,25)$.

The DUMC Clinical Microbiology Laboratory (CML) maintains a frozen sample $\left(-70^{\circ} \mathrm{C}\right)$ of all bacterial isolates obtained from blood as well as isolates obtained from respiratory specimens of persons with CF. Gram-negative, oxidase-positive, nonfermenting rods were preliminarily identified as Bcc by cell wall analysis with gas-liquid chromotography (MIDI System; Microbial ID, Newark, DE). The CML database was queried for all cultures submitted from this cohort of patients to identify cultures that grew Bcc. Isolates were categorized based on clinical specimen type and date of specimen collection and those isolates available were recovered from frozen stocks and further analyzed at the Burkholderia cepacia Research Laboratory and Repository, University of Michigan. Isolates were confirmed as members of the genus Burkholderia, and species designation within the Bcc was determined utilizing $16 \mathrm{~S}$ rDNA and recA species-specific polymerase chain reaction (PCR) and recA restriction fragment length polymorphism (RFLP) analyses $(26,27)$. Box PCR typing was performed, as previously described, on isolates to determine strain relatedness (28). For the purposes of this study, distinct strain types were assigned different alphabetic designations.

\section{Statistical method}

Statistical analyses were performed using Analyse-lt software (AnalyseIt Software Ltd, Leeds, UK) for Microsoft Excel and JMP Statistical Discovery Program (version 6.0, SAS Institute, Cary, NC) and a type-I error rate of 0.05 . Descriptive statistics performed on the data set included measures of central tendency and spread for continuous variables and percentages and frequencies for categorical variables. Infection status within 1 year prior to transplant was divided into three following categories for analysis purpose: patients without $\mathrm{Bcc}$ isolated were considered 'noninfected', patients harboring a species of Bcc other than B. cenocepacia were considered 'other Bcc species' and patients harboring B. cenocepacia were considered 'B. cenocepacia'. Subjects for whom Bcc was isolated within 1 year of LT but for whom no isolate was available for further speciation were excluded from analysis. Subjects for whom Bcc was not isolated prior to transplant but was isolated after transplant were considered 'noninfected'. Three way comparisons between the groups based on infection status were performed using oneway ANOVA for continuous variables including the Kruskal-Wallis test for nonparametric sensitivity analyses and Tukey's method for multiple comparisons. The chi-square test was used for categorical variables. Survival curves were calculated with the Kaplan-Meier method and compared using the log-rank test. Survival status was divided into two categories-'alive' or 'dead or retransplanted' at 1 year post LT. Logistic regression analyses were performed to investigate the relationship of infection status, recipient age at time of transplant, donor age, mechanical ventilation for recipient at time of LT, graft ischemia time, mismatches in human leukocyte antigens, receipt of trimethoprim/sulfamethoxazole following transplant and donor/recipient CMV serostatus on survival status 1 year post LT. The sample size was insufficient for multivariable modeling. Missing data points were not replaced.

\section{Results}

Seventy-six consecutive CF patients underwent LT at DUMC between September 1992 and August 2002. During the year before transplant, 59 (77.6\%) patients were 'noninfected', 9 (12.0\%) harbored 'other Bcc species', including $B$. multivorans (7 patients, $9.2 \%$ ) and $B$. vietnamiensis (2 patients, $2.6 \%$ ), and $7(9.2 \%)$ harbored B. cenocepacia. One patient was excluded because her pretransplant isolate was not available for evaluation. Two patients had $B$. cenocepacia isolated post transplant, including one patient with both $B$. cenocepacia and B. multivorans, but no Bcc isolated pretransplant. These patients were included in the 'noninfected' group for primary analysis. Four patients required a second transplant. 
Patient characteristics at the time of lung transplantation are included in Table 1. The average patient age was 27.7 years (SD 8.5, range 11 to 53). Gender, CMV serostatus, mean age at time of transplant, mean donor age, median graft ischemic time and number of HLA mismatches did not differ significantly between the three groups. All patients were Caucasian and only one, who was infected with B. cenocepacia, was of Hispanic/Latino ethnicity. Six (86\%) patients had multidrug resistant $B$. cenocepacia, of which three (43\%) had panresistant B. cenocepacia. Seven (78\%) patients infected with 'other Bcc species' had multidrug resistant organisms recovered, of which five (56\%) were considered panresistant. Similarly, five (71\%) and seven $(78 \%)$ patients in the B. cenocepacia and 'other Bcc species' groups, respectively, were colonized with other potential pathogens including Staphylococcus aureus or another gram-negative rod. These differences were not significant.

Of 16 CF patients infected with Bcc prior to transplant, all but two had Bcc recovered from respiratory cultures post transplant. These two patients had 9 and 4 BAL samples cultured post transplant, respectively. All patients infected with Bcc prior to transplant and who had Bcc recovered from clinical specimens post transplant retained their pretransplant strains (Table 2).

Within 1 year of LT, 11 patients died including $8.5 \%$ (5/59) of patients in the 'noninfected' group, $11.1 \%$ (1/9) of patients in the 'other Bcc species' group and $71.4 \%$ (5/7) of patients in the ' $B$. cenocepacia' group. Infection status was significantly associated with 1 -year survival ( $<<0.0001)$. Survival estimates for 'noninfected' patients were 97\%, 92\%, 76\% and $63 \%$, at 1 month, 1 year, 3 years and 5 years, respectively, compared with $89 \%, 89 \%, 67 \%$ and $56 \%$ in patients with 'other Bcc species' and 71\%, 29\%, 29\% and $29 \%$ for patients with 'B. cenocepacia' ( $p=0.0142$, Figure 1). Survival estimates were worse for patients with 'B. cenocepacia' when compared with all other patients combined as a single group ( $p<0.0037)$. In fact, patients infected with $B$. cenocepacia prior to transplant were eight times more likely to die within 1 year of transplant than those not infected $(R R=8.43,95 \% \mathrm{Cl} 3.23-22.03, \mathrm{p}<$ $0.00005)$ and six times more likely to die within 1 year of transplant than those infected with 'other Bcc species' prior to transplant $(R R=6.43,95 \% \mathrm{Cl}, 0.95-43.26$, $\mathrm{p}=0.04)$.

Gender, recipient age, donor age, mechanical ventilation at time of transplant, graft ischemic time, mismatches in human leukocyte antigens, CMV serostatus, receipt of trimethoprim/sulfamethoxazole following transplant and pre LT infection with 'other Bcc species' had no association with 1-year survival.

\section{Discussion}

Several studies have established that for CF patients, infection with Bcc is an independent predictor of early death and accelerated decline in pulmonary function. Thus, many patients infected with Bcc seek lung transplantation as a life-extending option when their respiratory status begins to decline. This investigation into the impact of Bcc

Table 1: Patient characteristics at the time of lung transplantation by pre-transplant Burkholderia cepacia complex (Bcc) infection status

\begin{tabular}{|c|c|c|c|}
\hline \multirow[b]{2}{*}{ Variable } & \multicolumn{3}{|c|}{ Infection status $^{1}$} \\
\hline & $\begin{array}{l}\text { B. cenocepacia } \\
\qquad N=7\end{array}$ & $\begin{array}{c}\text { Other Bcc species }{ }^{2} \\
\quad N=9\end{array}$ & $\begin{array}{l}\text { Noninfected } \\
N=59\end{array}$ \\
\hline Recipient age in years & 24 & 28 & 27 \\
\hline (Median, IQR) & $(21-33)$ & $(27-33)$ & $(22-33)$ \\
\hline Male N (\%) & $3(42.9)$ & $4(44.4)$ & $26(44.1)$ \\
\hline Mechanical ventilation at time of transplant N (\%) & 0 & 0 & $6(10.2)$ \\
\hline \multicolumn{4}{|l|}{$\mathrm{CMV}$ risk status ${ }^{3} \mathrm{~N}(\%)$} \\
\hline High & $2(28.6)$ & $3(33.3)$ & $19(32.2)$ \\
\hline Intermediate & $4(57.1)$ & $5(55.6)$ & $22(37.3)$ \\
\hline Low & $1(14.3)$ & $1(11.1)$ & $18(30.5)$ \\
\hline Donor age in years & 22 & 17 & 25 \\
\hline (Median, IQR) & $(17-28)$ & $(15-26)$ & $(15-40)$ \\
\hline $\begin{array}{l}\text { No. HLA mismatches } \\
\text { (Median, IQR) }\end{array}$ & $5(4-5.5)$ & $4(4-5)$ & $5(4-5.25)$ \\
\hline Graft ischemic time (h) & 634 & 652 & 636 \\
\hline (Median, IQR) & (601.5-713.5) & $(525-706)$ & $(547.5-728.8)$ \\
\hline
\end{tabular}


Alexander et al.

Table 2: Burkholderia cepacia complex species and strain type infecting CF patients prior to and 5 years after lung transplantation

\begin{tabular}{|c|c|c|c|c|c|}
\hline \multirow[b]{2}{*}{ Patient } & \multirow{2}{*}{$\begin{array}{l}\text { Burkholderia } \\
\text { species }\end{array}$} & \multicolumn{2}{|c|}{ Strain type ${ }^{1}$} & \multirow{2}{*}{$\begin{array}{c}\text { Survival } \\
\text { posttransplant (Days) }\end{array}$} & \multirow[b]{2}{*}{ Cause of death } \\
\hline & & Pretransplant & Posttransplant & & \\
\hline 1 & B. cenocepacia & AA & AA & 29 & Burkholderia pneumonia \\
\hline 2 & B. cenocepacia & I & No Bcc isolated & 16 & Anoxic brain injury \\
\hline 3 & B. cenocepacia & W & W & 170 & Burkholderia sepsis \\
\hline 4 & B. cenocepacia & I & 1 & $>1825$ & Alive \\
\hline 5 & B. cenocepacia & $x$ & No Bcc isolated & 266 & Respiratory failure/Rejection \\
\hline 6 & B. cenocepacia & $A F$ & $A F$ & $>1825$ & Alive \\
\hline 7 & B. cenocepacia & S & S & 158 & Burkholderia sepsis \\
\hline 8 & $\begin{array}{l}\text { B. cenocepacia } \\
\text { B. multivorans }\end{array}$ & No Bcc isolated & $\begin{array}{c}B \text { and } A \\
C\end{array}$ & 223 & Respiratory failure \\
\hline 9 & B. cenocepacia & No Bcc isolated & B & $>1825$ & Alive \\
\hline 10 & B. multivorans & $U$ & $U$ & 1820 & Burkholderia sepsis \\
\hline 11 & B. multivorans & $\mathrm{Al}$ & $\mathrm{Al}$ & 1086 & Cancer \\
\hline 12 & B. multivorans & $\mathrm{T}$ & $\mathrm{T}$ & $>1825$ & Alive \\
\hline 13 & B. multivorans & AJ & AJ & $>1825$ & Alive \\
\hline 14 & B. multivorans & $\mathrm{AH}$ & Bcc isolate not saved & $>1825$ & Alive \\
\hline 15 & B. multivorans & $\mathrm{L}$ & $\mathrm{L}$ & $>1825$ & Alive \\
\hline 16 & B. multivorans & $A C$ & $\mathrm{AC}$ & 1651 & Bronchiolitis obliterans syndrome \\
\hline 17 & B. vietnamiensis & $\mathrm{N}$ & $\mathrm{N}$ & 26 & Diffuse alveolar damage \\
\hline 18 & B. vietnamiensis & $A B$ & $A B$ & $>1825$ & Alive \\
\hline
\end{tabular}

${ }^{1}$ Each unique alphabet designation represents a distinct strain type, as determined by Box PCR typing.

respiratory infection in CF patients on re-infection rates and survival following lung transplantation offers additional and important insight for the management of these patients. Contrary to the early data from our institution, infection with Bcc prior to transplant, and more specifically, infection with $B$. cenocepacia, significantly impacts survival. Mortality was significantly worse for those CF patients infected with B. cenocepacia compared with noninfected CF patients and CF patients infected with Bcc species other than $B$ cenocepacia. Furthermore, the negative effect was most dramatic during the first year following the transplant pro- cedure. Per Table 2, over half of the patients infected with B. cenocepacia in our cohort were dead within the first 6 months following the transplant procedure, and Burkholderia pneumonia or sepsis was considered the cause of death for all but one. In contrast, Burkholderia infection was considered the cause of death for only one patient infected with other Burkholderia species and as a contributor to the cause of death for only one patient not infected with Bcc prior to transplant (acquired following the transplant procedure), during the 5-year follow-up. Although this study is clearly limited by the small number of patients with

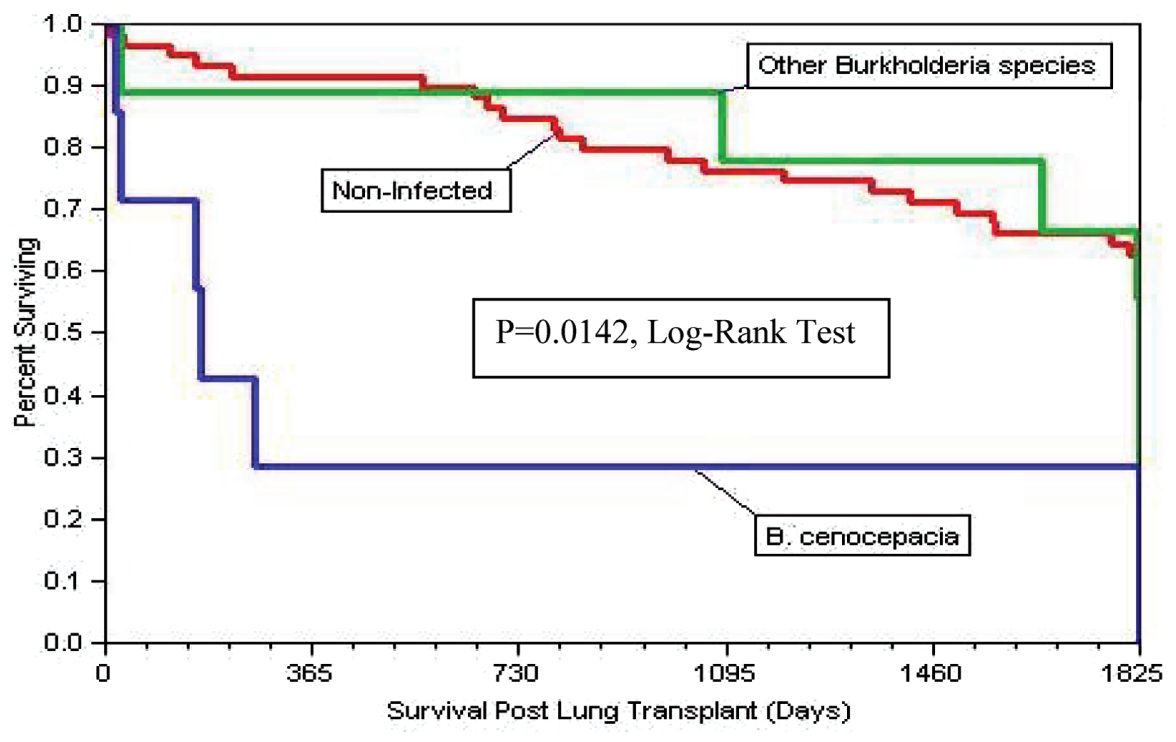

Figure 1: Five-year survival for cystic fibrosis patients following lung transplant based on pretransplant Burkholderia cepacia complex infection status. Infection status within 1 year prior to transplant was divided into three categories for analysis purposes as follows: patients without Bcc isolated were considered 'noninfected', patients harboring a species of Bcc other than $B$. cenocepacia were considered 'other Burkholderia species' and patients harboring $B$. cenocepacia were considered ' $B$. cenocepacia'. 
$B$. cenocepacia in the cohort and the retrospective design, the results are striking and are consistent with reports from other LT centers $(21,22)$. For example, De Soyza and coworkers in Newcastle-Upon-Tyne, UK, reported early deaths following lung transplantation for 4 of 4 patients infected with $B$. cenocepacia strain ET12, which is common in eastern Canada and the UK and has been associated with poor outcome (29), but no deaths among 5 patients infected with other Bcc species (mean follow-up 1.9 years) (21). Aris and colleagues at the University of North Carolina reported the death of 5 of 12 patients infected with $B$. cenocepacia (none of which were infected with strain ET12) compared with no deaths in the 8 patients infected with other Bcc species (mean follow-up 1.5 years) (22). Our data confirm the early mortality associated with $B$. cenocepacia infection and offer important new insights into the long-term survival of patients infected with other Bcc species. In fact, 5-year survival for patients infected with Bcc other than B. cenocepacia did not significantly differ from that of noninfected CF patients and exceeds that of other groups considered at high-risk following lung transplantation, such as those transplanted due to idiopathic pulmonary fibrosis or primary pulmonary hypertension (www.ustransplant.org/annual_reports/current/ survival_rates.htm). As with the study by Aris et al., the heterogeneity of $B$. cenocepacia strains recovered in our cohort also indicates that increased virulence is not limited to a single strain type (e.g. B. cenocepacia strain ET12). However, whether these observations can be extrapolated to conclude that 'all' strains of $B$. cenocepacia have the same capacity for poor outcome in LT recipients is not clear.

Consistent with previous reports from other institutions, CF patients in our cohort were almost uniformly re-infected with their pretransplant infecting strain of Bcc following the LT procedure (14). All transplant recipients in this cohort received bilateral LTs, thereby eliminating a native lung as a potential source of re-infection. All transplanted grafts were cultured before implantation and none grew Bcc, effectively ruling out the transplanted lungs as the source of infection. The sinuses are another potential and highly likely reservoir for autologous infection of the new lungs. Whether prophylactic sinus procedures in CF patients prior to transplant would decrease the rate of pulmonary reinfection would need to be studied. The majority of patients in our cohort did not undergo sinus surgery within 1 year prior to transplant; only one patient colonized with 'B. cenocepacia' and one patient colonized with 'other Bcc species' underwent sinus surgery. Another important potential source for re-infection is the patient's caregivers; although if this were the case, one would expect re-infection with a strain different from their pretransplant clone in at least some of the patients. With that said, our data do highlight the importance of stringent infection control practices to decrease nosocomial transmission of these pathogens. Two of our patients had no $B$. cenocepacia recovered before transplantation, but did develop infection with $B$. cenocepacia post transplant. One of these patients had B. ceno- cepacia first recovered from BAL collected 70 days post transplant although 5 total respiratory samples collected throughout the year preceding transplant and the first 30 BALs collected post transplant did not grow a Bcc species. Strain typing revealed this and subsequent $B$. cenocepacia strains ( $B$ and $A$ ) recovered from this patient to be the two strains identified during an outbreak of $B$. cenocepacia bloodstream infections at our institution between August 1997 and April 2001 (13). This patient also had B. multivorans recovered for the first time from BAL collected 85 days post transplant. Interestingly, this patient was never discharged following transplant, resulting in a 7-month hospitalization. The second patient had $B$. cenocepacia recovered from respiratory samples 2 months following transplant. Box PCR typing revealed this isolate to also be one of the B. cenocepacia institutional outbreak strains (B). Given the enhanced capacity for interpatient spread and associated poor outcome, our center instituted strict infection control measures, including gowning and gloving upon entering the room of and handwashing after contact with all Bcc-infected patients. The frequency of Bcc recovery within the institution rapidly decreased after these interventions. Accordingly, in addition to adherence to routine infection control practices for all patients undergoing lung transplantation, we strongly recommend placing any patient infected with $B$ cenocepacia under strict isolation precautions (13).

Due to the excessive mortality seen among patients infected with $B$. cenocepacia, the LT community is beginning to re-consider lung transplantation as an option for patients infected with this species. Available data support screening of all CF patients for Bcc and excluding those infected with $B$. cenocepacia from transplantation. Active research continues regarding pathogenesis and epidemiology of Bcc among CF patients. Currently, International Society for Heart and Lung Transplantation guidelines do not list $B$. cenocepacia infection as an absolute contraindication to lung transplantation; however, 'colonization with highly resistant or highly virulent bacteria' is included as a relative contraindication, thereby giving individual centers the ability to dictate practice (30). As with other reports, for CF patients infected with Bcc, antibiotic susceptibility patterns were not helpful in determining the likelihood of survival following lung transplantation (22). Although the optimization of antibiotic combinations and attenuation of immunosuppression for patients infected with Bcc have been offered as means of improving survival for these patients following transplant (17), careful selection of a 4drug antibiotic regimen was not found to be beneficial in our group of patients with $B$. cenocepacia. Similarly, treatment with trimethoprim-sulfamethoxazole did not impact outcomes. Furthermore, given the very high rates of acute and chronic rejection after lung transplantation, it is unclear if a strategy of reduced immunosuppression would lead to any better long-term outcomes in this population due to the potential for a corresponding increase in rejection related deaths. 
Cooperation between CF treatment and LT centers will hopefully provide new insights into virulence, transmissibility and treatment regimens for this unique and challenging pathogen. More specifically, further studies to identify which specific strains of $B$. cenocepacia may be more virulent, the mechanisms behind the virulence in such strains and investigations to tease out what host factors might influence progression of the infection in the CF population should be a priority. Until then, we recommend the careful screening of all CF patients for Bcc and excluding from LT those harboring $B$. cenocepacia, regardless of susceptibility profile.

\section{Acknowledgment}

This study was funded in part by the Cystic Fibrosis Foundation.

\section{References}

1. Coenye T, Vandamme P, Govan JR, LiPuma JJ. Taxonomy and identification of the Burkholderia cepacia complex. J Clin Microbiol 2001; 39: 3427-3436.

2. LiPuma JJ. Burkholderia and emerging pathogens in cystic fibrosis. Semin Respir Crit Care Med 2003; 24: 681-692.

3. Vandamme P, Holmes B, Vancanneyt M et al. Occurrence of multiple genomovars of Burkholderia cepacia in cystic fibrosis patients and proposal of Burkholderia multivorans sp. nov. Int J Syst Bacteriol 1997; 47: 1188-1200.

4. Vandamme P, Henry D, Coenye T et al. Burkholderia anthina sp. nov. and Burkholderia pyrrocinia, two additional Burkholderia cepacia complex bacteria, may confound results of new molecular diagnostic tools. FEMS Immunol Med Microbiol 2002; 33: 143-149.

5. Isles A, Maclusky I, Corey M et al. Pseudomonas cepacia infection in cystic fibrosis: an emerging problem. J Pediatr 1984; 104: 206210.

6. Tablan OC, Martone WJ, Doershuk CF et al. Colonization of the respiratory tract with Pseudomonas cepacia in cystic fibrosis. Risk factors and outcomes. Chest 1987; 91: 527-532.

7. Courtney JM, Dunbar KE, McDowell A et al. Clinical outcome of Burkholderia cepacia complex infection in cystic fibrosis adults. J Cyst Fibros 2004; 3: 93-98

8. Reik R, Spilker T, LiPuma JJ. Distribution of Burkholderia cepacia complex species among isolates recovered from persons with or without cystic fibrosis. J Clin Microbiol 2005; 43: 2926-2928.

9. LiPuma JJ, Spilker T, Gill LH, Campbell PW III, Liu L, Mahenthiralingam E. Disproportionate distribution of Burkholderia cepacia complex species and transmissibility markers in cystic fibrosis. Am J Respir Crit Care Med 2001; 164: 92-96.

10. Speert DP, Henry D, Vandamme P, Corey M, Mahenthiralingam E. Epidemiology of Burkholderia cepacia complex in patients with cystic fibrosis, Canada. Emerg Infect Dis 2002; 8: 181-187.

11. Agodi A, Mahenthiralingam E, Barchitta M, Giannino V, Sciacca A, Stefani S. Burkholderia cepacia complex infection in Italian patients with cystic fibrosis: Prevalence, epidemiology, and genomovar status. J Clin Microbiol 2001; 39: 2891-2896.

12. LiPuma JJ. Burkholderia cepacia. Management issues and new insights. Clin Chest Med 2000; 19: 473-486.

13. Woods CW, Bressler AM, LiPuma JJ et al. Virulence associated with outbreak-related strains of Burkholderia cepacia complex among a cohort of patients with bacteremia. Clin Infect Dis 2004; 38: $1243-1250$.
14. Steinbach S, Sun L, Jiang RZ et al. Transmissibility of Pseudomonas cepacia infection in clinic patients and lung-transplant recipients with cystic fibrosis. N Engl J Med 1994; 331: 981-987.

15. Aris RM, Gilligan PH, Neuringer IP, Gott KK, Rea J, Yankaskas JR. The effects of panresistant bacteria in cystic fibrosis patients on lung transplant outcome. Am J Respir Crit Care Med 1997; 155: 1699-1704.

16. Snell GI, de Hoyos A, Krajden M, Winton T, Maurer JR. Pseudomonas cepacia in lung transplant recipients with cystic fibrosis. Chest 1993; 103: 466-471.

17. Chaparro C, Maurer J, Gutierrez C et al. Infection with Burkholderia cepacia in cystic fibrosis: Outcome following lung transplantation. Am J Respir Crit Care Med 2001; 163: 43-48.

18. Kanj SS, Tapson V, Davis RD, Madden J, Browning I. Infections in patients with cystic fibrosis following lung transplantation. Chest 1997; 112: 924-930.

19. Egan JJ, Chadwick P, Lowe L, Woodcock AA. The potential of nosocomial transmission of Pseudomonas cepacia exists at cardiopulmonary transplant centers. Chest 1994; 105: 16301631.

20. Flume PA, Egan TM, Paradowski LJ, Detterbeck FC, Thompson JT, Yankaskas JR. Infectious complications of lung transplantation. Impact of cystic fibrosis. Am J Respir Crit Care Med 1994; 149 : 1601-1607.

21. De Soyza A, McDowell A, Archer L et al. Burkholderia cepacia complex genomovars and pulmonary transplantation outcomes in patients with cystic fibrosis. Lancet 2001; 358: 17801781.

22. Aris RM, Routh JC, LiPuma JJ, Heath DG, Gilligan PH. Lung transplantation for cystic fibrosis patients with Burkholderia cepacia complex. Survival linked to genomovar type. Am J Respir Crit Care Med 2001; 164: 2102-2106.

23. Mahenthiralingam E, Vandamme $P$, Campbell ME et al. Infection with Burkholderia cepacia complex genomovars in patients with cystic fibrosis: Virulent transmissible strains of genomovar III can replace Burkholderia multivorans. Clin Infect Dis 2001; 33: 1469 1475.

24. Hadjiliadis D, Howell DN, Davis RD et al. Anastomotic infections in lung transplant recipients. Ann Transplant 2000; 5: 13-19.

25. Palmer SM, Perfect JR, Howell DN et al. Candidal anastomotic infection in lung transplant recipients: Successful treatment with a combination of systemic and inhaled antifungal agents. J Heart Lung Transplant 1998; 17: 1029-1033.

26. LiPuma JJ, Dulaney BJ, McMenamin JD et al. Development of rRNA-based PCR assays for identification of Burkholderia cepacia complex isolates recovered from cystic fibrosis patients. J Clin Microbiol 1999; 37: 3167-3170.

27. Mahenthiralingam E, Bischof J, Byrne SK et al. DNA-Based diagnostic approaches for identification of Burkholderia cepacia complex, Burkholderia vietnamiensis, Burkholderia multivorans, Burkholderia stabilis, and Burkholderia cepacia genomovars I and III. J Clin Microbiol 2000; 38: 3165-3173.

28. Coenye T, Spilker T, Martin A, LiPuma JJ. Comparative assessment of genotyping methods for epidemiologic study of Burkholderia cepacia genomovar III. J Clin Microbiol 2002; 40: 3300-3307.

29. Ledson MJ, Gallagher MJ, Jackson M, Hart CA, Walshaw MJ. Outcome of Burkholderia cepacia colonisation in an adult cystic fibrosis centre. Thorax 2002; 57: 142-145.

30. Orens JB, Estenne M, Arcasoy S et al. International guidelines for the selection of lung transplant candidates: 2006 update-A consensus report from the Pulmonary Scientific Council of the International Society for Heart and Lung Transplantation. J Heart Lung Transplant 2006; 25: 745-755. 